\title{
Editorial
}

\section{Hepatology in the COVID Era: Another C Virus, again Challenging the Liver}

\author{
Paula Peixe $^{a}$ Filipe Calinas $^{b}$ Rui Tato Marinho ${ }^{c}$ \\ ${ }^{a}$ Gastroenterology Department, Centro Hospitalar de Lisboa Ocidental, epe, Lisboa, Portugal; ${ }^{b}$ Gastroenterology \\ Department, Centro Hospitalar de Lisboa Central, epe, Lisboa, Portugal; ' 'Gastroenterology Department, Centro \\ Hospitalar de Lisboa Norte, epe, Lisboa, Portugal
}

Keywords

Liver · Cirrhosis · Coronavirus · COVID-19

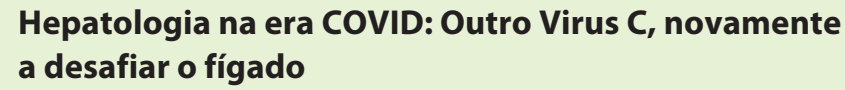

\section{Palavras Chave}

Fígado $\cdot$ Cirrose $\cdot$ Coronavirus · COVID-19

Our way of life has changed! The world is changing dramatically. Read the story! [1].

Since the beginning of 2020 , the worldwide spread of a new virus has made us rethink and reinvent new ways of living and caring for patients. Doctors no longer have "the magic touch."

In late 2019, a new type of severe pneumonia associated with a new type of coronavirus was first detected in the Chinese province of Hubei (People's Republic of China).

Coronaviruses have existed for a long time, conditioning diseases in several animal species, but until 2003

\section{KARGER}

karger@karger.com www.karger.com/pjg
(C) 2020 Sociedade Portuguesa de Gastrenterologia Published by S. Karger AG, Basel

Karger

Upen access

This article is licensed under the Creative Commons AttributionNonCommercial-NoDerivatives 4.0 International License (CC BYNC-ND) (http://www.karger.com/Services/OpenAccessLicense). Usage and distribution for commercial purposes as well as any distribution of modified material requires written permission.
(SARS [Severe Acute Respiratory Syndrome]) there was no report of transmission to humans.

The new coronavirus, initially called $2019 n-\operatorname{Cov}$ and now SARS-Cov2, is the seventh coronavirus to infect humans, as identified by the Chinese health authorities.

It emerged as a human-transmitted zoonosis originating from the wild animal market in the city of Wuhan. Viruses with similar characteristics have been identified in bats, snakes, and pangolins, with an homology of genomic features showing an 89 and $82 \%$ nuclear acid sequence similarity with bat SARS-CoVZXC21 and human SARS-CoV (Wuhan patients) but the true transmitter of the disease has not yet been found [2].

The disease is called COVID-19 (COrona VIrus Disease 2019, i.e., the year of the first case and virus identification). From the point of view of transmission, it occurs among humans through contamination by particles expelled through the airways (by coughing, sneezing, and talking) but also by fomites and interpersonal contact. Aerosols and fecal-oral transmission are also considered potential sources of contamination. In addition to this community transmission, nosocomial transmission is important, since many symptomatic patients use health services where they can infect other people, particularly 
health professionals, when not adequately protected. The risk of infection for health professionals is 3 times more than in the general population [3].

The spread of COVID-19 occurred in the beginning by contact with the original cases identified in Wuhan but then, within $<30$ days, it went from reaching a city to the whole country. The Chinese New Year season had a decisive impact as it corresponds to a period of the year when thousands of people are travelling. Public transport, always full, favored the transmission of the infection at this time [4]. Due to the ease of travel nowadays, cases quickly spread to all countries in the world. Around 40 million flights worldwide each year is a terrific number for the capacity of disease transmission.

It was possible to designate the transmission chain by identifying index cases. However, in each country, transmission started to occur locally and the links were lost. By March 2020, COVID-19 had become widespread in all countries and the World Health Organization declared it a pandemic. Today, about $2,400,000$ cases and 165,000 deaths were reported worldwide (https://www.worldometers.info/coronavirus/ [accessed 2020abril19 18:00]).

\section{The Virus Is Attacking Us}

Understanding the pathophysiology of viral infection and the relationship between virus and host has become a matter of urgency. The scientific community has made an intense effort to identify these relationships, but most of the issues are still open. It was possible to identify the virus receptor in humans, namely, the angiotensin-converting enzyme 2 (ACE2), a transcellular protein to which SARS-Cov2 binds. The cellular serine protease, transmembrane protease serine 2 (TMPRSS2), which cleaves the $S$ protein of human coronaviruses onto the cell membrane, is critical for the fusion of viral cells and the cellular membranes in the host.

The coexpression of these molecules seems to be crucial to the infection. ACE2 is widely expressed in the human body, i.e., in the lungs, heart, vasculature, kidneys, brain, intestines, and testes. It is also expressed in hepatocytes and in cholangiocytes. The same is true for TMPRSS2 [5-7].

The ubiquity of distribution of ACE2 and TMPRSS2 may explain the systemic involvement of the SARS-Cov2 infection, however, the disease activity is more significant at the respiratory level due to the expression of the molecules in these organs. Patients may remain asymptomatic, without knowing exactly the percentage of infected people who do not have manifestations of the disease [8]. Respiratory conditions manifesting as fever (41\%), cough (56\%), headache (25\%), muscle pain (29\%), and generalized weakness (24\%) can have a mild and moderate evolution. Severe cases with respiratory insufficiency eventually require ventilatory support (and, in some cases, extracorporeal life support); this has occurred in Portugal in $17 \%$ of cases according to the records $[9,10]$.

Gastrointestinal (GI) manifestation in COVID-19 patients who report at least one GI tract symptom (nausea, vomiting, or diarrhea) varies from 5 to $11.4 \%$. Jin et al. [11] reported a rate of chronic liver disease of $10.81 \%$ in patients with GI symptoms, significantly higher than those without these symptoms $(2.95 \%)(p=0.004)$. They also reported that the rate of COVID-19 patients with severe disease is significantly higher in those with GI symptoms $(22.97$ vs. $8.14 \%, p<0.001)$.

\section{The Impact of COVID-19 on the Liver}

The impact of COVID-19 on the liver is unclear. The analysis of this relationship must be done by examining 2 distinct aspects, namely liver damage caused by the new coronavirus/COVID-19 and the impact of preexisting liver disease on COVID-19.

\section{Liver Damage Caused by COVID-19}

As already mentioned, the molecules involved in infectivity of the virus are also present in the cells of the liver (hepatocytes and cholangiocytes), so the liver is damaged by SARS-Cov2, as occurs with the other coronaviruses.

The initial description of hepatic involvement emerged from the analysis of the first 99 cases, 43 of whom presented with an elevation of liver enzymes, alanine aminotransferase in $28 \%$ of patients, aspartate aminotransferase in $35 \%$, and total bilirubin in $18 \%$.

The incidence of elevated serum liver biochemistry in hospitalized patients with COVID-19 ranges from 14 to $53 \%$. In a recent review, changes in liver test values were recorded in about $46 \%$ of patients at diagnosis (Table 1) and more frequently during hospitalization (Table 2) [12]. These changes were mild to moderate. Only 1 patient had a significant hepatocellular lesion. There was no identification of a cholestatic pattern or liver failure [13]. Zhan et al. (unpubl. data from their own center), reported the presence of elevated $\gamma$-glutamyl transferase (GGT) in 30 (54\%) of 56 patients with COVID-19 during hospitalization. Such data have not been previously recorded [14]. 
Table 1. Liver tests of COVID-19 patients at diagnosis

\begin{tabular}{llll}
\hline & \multirow{2}{*}{ Patients } & \multicolumn{2}{l}{ Laboratory data } \\
\cline { 3 - 4 } & & normal \% $(n)$ & abnormal \% $(n)$ \\
\hline Liver tests & 417 & $54(225)$ & $46(195)$ \\
ALT & 417 & $87(363)$ & $13(54)$ \\
AST & 417 & $82(341)$ & $18(76)$ \\
GGT & 417 & $84(349)$ & $16(68)$ \\
ALP & 332 & $95(316)$ & $5(16)$ \\
TBIL & 414 & $77(318)$ & $23(96)$ \\
\end{tabular}

ALT, alanine aminotransferase; AST, aspartate transaminase; GGT, $\gamma$-glutamyl-transpeptidase; ALP, alkaline phosphatase; TBIL, total bilirubin [12].

Recently, an association between the severity of COVID-19 and the alteration of liver tests has been described, showing that patients with more severe or critical disease have significantly higher aminotransferase and bilirubin values than cases of mild-to-moderate disease. Hypoalbuminemia and low platelets are also associated with more serious disease, but may be related to COVID-19 instead of previous liver disease.

Following autopsy studies, liver changes included dark red hepatomegaly, hepatocyte degeneration with focal lobular necrosis and infiltration by neutrophils, infiltration by lymphocytes and monocytes in the portal spaces, and sinusoidal congestion with microthrombosis. Despite these findings, no lesions of the bile ducts or liver failure characteristics were identified [15].

Multiple mechanisms involved in liver injury have been described: immune-mediated damage, direct cytotoxicity, anoxia due to respiratory failure, drug-induced liver injury, and the reactivation of preexisting liver disease [16]. After recovery, liver enzyme values return to normal and no evolutive disease seems to occur.

The Impact of Preexisting Liver Disease on COVID-19

Chronic liver disease has a significant burden worldwide that can be assessed by the joint contribution of cirrhosis (11th) and liver cancer (16th) as the cause of 3.5\% of all deaths [17]. In published series, liver disease was not identified as a risk factor for SARS-Cov2 infection [1115]. It is assumed that risk factors for the general population are also risk factors for patients with liver disease. However, COVID-19 may indeed be more severe in liver patients.

Hepatic disease is associated with multiple factors including viral diseases, alcoholic disease, non-alcoholic
Table 2. Liver tests of COVID-19 patients at hospitalization

\begin{tabular}{llll}
\hline & Patients, $n$ & \multicolumn{2}{c}{ Laboratory data } \\
\cline { 3 - 3 } & & normal \% $(n)$ & abnormal \% $(n)$ \\
\hline Liver tests & 318 & $7(21)$ & $93(297)$ \\
ALT & 318 & $41(131)$ & $59(187)$ \\
AST & 318 & $53(168)$ & $47(150)$ \\
GGT & 318 & $51(163)$ & $49(155)$ \\
ALP & 283 & $89(252)$ & $11(31)$ \\
TBIL & 318 & $36(114)$ & $64(204)$ \\
\hline
\end{tabular}

ALT, alanine aminotransferase; AST, aspartate transaminase; GGT, $\gamma$-glutamyl-transpeptidase; ALP, alkaline phosphatase; TBIL, total bilirubin [12].

fatty liver disease (NAFLD), and autoimmune pathology (particularly autoimmune hepatitis and primary biliary cholangitis and other cholangiopathies). Special reference must be made to cirrhosis, hepatocellular carcinoma (HCC), and transplantation.

Despite this significant burden, the impact of COVID-19 on liver disease and liver disease on COVID-19 has not been evaluated and studies are needed to better understand the impact of these associations. Despite this, some situations deserve a comment for the lessons that its analysis can give.

The COVID-19 pandemic started in China and rapidly spread to nearby countries. Southeast Asia has a high prevalence of hepatitis $B$ virus (HBV) infection. In the study by Guan et al. [15] 23 patients $(n=1,099)$ had HBV, according to positive tests for HBV surface antigen (HBs $\mathrm{Ag}$ ) with or without elevated liver enzymes. Of these 23 patients, 22 had nonsevere disease (2.4 vs. $0.6 \%)$. No warning signs were triggered by the association of these infections.

No reference has yet been made to hepatitis $C$ virus (HCV) in the literature nor any comment has come from the USA. In the USA, the country with the largest number of COVID-19 cases and with a relevant expression of hepatitis $\mathrm{C}$, no statement has been issued by the Centers of Disease Control and Prevention or any group under surveillance, e.g., the Veterans Cohort. At this stage, it is urgent to modify the strategy regarding the approach to $\mathrm{HCV}$, simplifying the necessary assessments for the institution of treatment and its availability.

Approximately 2 billion adults are obese or overweight and over 400 million have diabetes. These are risk factors for NAFLD and HCC [17]. Diabetes, hypertension, and obesity associated with NAFLD are also de- 
scribed as factors for a poor prognosis associated with COVID-19.

A preliminary report on NAFLD in patients with COVID-19 calls attention to an increased risk of disease progression [6.6\% (5/126) vs. $44.7 \%(34 / 76) p<0.0001]$, a higher probability of abnormal liver function on admission and at discharge $[70 \%(53 / 76)$ vs. $11.1 \%(14 / 126) p<$ $0.0001]$, and a longer time for viral clearance (17.5 \pm 5.2 days vs. $12.1 \pm 4.4$ days $p<0.0001$ ), when compared with non-NAFLD individuals [18]. The authors state that NAFLD patients also had a higher risk of progression to severe COVID-19 and present an increased viral clearance time. With the global prevalence of NAFLD on the increase, it is worth noting that a significant number of patients may be at risk of developing severe COVID-19.

Immune-mediated liver diseases, particularly autoimmune hepatitis, have not been mentioned as risk factors for COVID-19, but the immunosuppressive treatment required has triggered fears about the risk of infection in patients. The analysis of previous outbreaks of human infection by coronaviruses (SARS 2002 and MERS 2012) did not record an increase in cases in patients under immunosuppression, or that when infected, the disease was more severe. Recent data from the red zones in China and Bergamo (Italy) have shown neither an increase in the number of cases nor in their severity in immunosuppressed patients. Even when infected, as long as there were no other risk factors, these patients had a mild or moderate course of COVID-19 [19].

No analysis of the different types of drugs used in immunosuppression in liver diseases has been carried out. A warning has been issued about the use of corticosteroids in the context of SARS-Cov2 infection, where the most seriously ill patients most likely need corticosteroid therapy. The use of these drugs is associated with increased mortality from coronavirus pneumonia. No assessment has been made in patients with liver disease who were previously on stable treatment with corticosteroids and were infected; however, it is assumed that low doses have no impact on COVID-19 (as described for patients in the Wuhan observational study) [20,21].

Lleo et al. [22] drew attention to two relevant points: balancing the need to maintain treatment to prevent a serious episode that may require therapeutic measures and hospitalization (with an increased risk of nosocomial infection) and assessing the need for certain procedures, e.g., postponing liver biopsy until a period of less epidemic risk.

There has been an expectation of canalicular lesions being caused by SARS-Cov2 due to the presence of ACE2 in cholangiocytes. However, recent research has shown that ACE2 has a canalicular specificity, which could explain the scarce documentation of the cholestatic lesions and the low number of cases in which GGT changes have been reported $[13,14]$. The impact of SARS-Cov2 infection on cholestatic diseases such as primary biliary cholangitis or sclerosing cholangitis is unknown; further studies are needed to understand whether there is any clinical relevance $[23,24]$.

Changes in liver tests, often associated with coagulation disorder and hypoalbuminemia, are commonly seen in patients with severe COVID-19. These changes fluctuate according to the severity of the respiratory disease (pneumonia) and are not due to the direct effect of the virus on the liver. This may explain why there was no worse prognosis in a group of patients with cirrhosis and SARS-Cov2 infection.

In a review of 7 published studies, it was possible to identify 42 patients with cirrhosis with mortality ranging from $0-2 \%$ [13]. Despite this strange benign report, doctors should consider patients with chronic liver disease, liver cirrhosis, or liver cancer, to be a risk group as they have underlying immune dysfunction and a more reserved prognosis than other patients that go on to develop acute respiratory failure syndrome. For these reasons, patients with chronic liver disease need particular attention and have different specificities from other patients, especially if they are elderly and/or have comorbidities.

Data on SARS-Cov2 infection in cirrhotic and HCC patients are scarce. Extensive records and targeted studies are needed to explore multiple open-ended questions such as the severity and mortality of COVID-19 and episodes of acute-on-chronic or decompensation associated with the presence of this disease (ascites, hepatic encephalopathy, digestive bleeding, kidney dysfunction, and the risk of infection) or the response to treatment $[25,26]$.

Regarding transplantation and cancer patients, there are also no warnings [16]. However, it is not yet possible to say whether transplantation-associated immunosuppression can alter the predisposition for the acquisition of SARS-Cov2 infection or how COVID-19 evolves in these patients. A small published series showed a mortality rate of $27.8 \%(5 / 18)$ in a mixed group of solid organ-transplanted patients (liver recipients $(33.3 \%$ [6/18]) infected with SARS-Cov2 [27]. Cancer patients undergoing chemotherapy may be at increased risk for illness and serious illness, especially if they have other risk factors.

However, it is not known whether patients with liver cancer are at the same risk. There are still no data on decompensated cirrhosis, patients on the transplantation 
list and, patients in the immediate posttransplant period or with rejection. Decisions about transplantation should be considered on a case-by-case basis at this stage of the pandemic [28].

Another concern is for patients with liver disease (either advanced or decompensated), namely, the use of drugs used off-label which have not been studied for use for the current indication or in fragile populations such as liver patients. Multiple drugs have been referenced and are under study, but currently there is no known effective treatment [29].

\section{Health Services and Facilities}

During the COVID-19 pandemic, recommendations have been issued to mitigate the transmission of SARSCov2, voluntarily isolation has been promoted, and frequenting public places has been advised against. Patients associate hospitals and clinics with the risk of transmitting the virus. Many patients have conditions that remain unchecked. However, it is necessary for patients to maintain regular follow-up and for situations that require intervention to be detected in a timely manner.

On the other hand, the risk-versus-benefit of some interventions must also be weighed up and those without a negative impact should be postponed. A careful assessment will need to be made of the risk of episodes requiring hospitalization and the consequences associated with it or the risk of increased mortality if patients do not receive appropriate treatment or necessary treatment is delayed.

Scientific societies for the study of the liver, alone or in association, have been publishing guidelines for the approach and management of liver patients. Basically, we can divide patients into outpatients and those patients who require hospitalization. Stable outpatients with compensated liver disease should be supported in order to maintain the prescribed medication and recognize early warning signs and promote health literacy. Teleconsultations are encouraged and consideration is given to carrying out diagnostic and follow-up exams.

It is proposed that the blood samples and ultrasound examinations should, if possible, be postponed. If they are deemed necessary, they should be carried out in laboratories nearby avoiding the patient's movement, so as to minimize contacts and the risk of SARS-Cov2 transmission. Patients must comply with all the protection and risk minimization rules proposed for the general population.
The maintenance of surveillance of HCC has been discussed. In high-risk patients, this must be maintained so that lesions that could have an early diagnosis are not detected too late.

Patients with decompensated liver disease should minimize their contact with health-care personnel and, if possible, should not be admitted to hospital. If a patient (without COVID-19) does have to be admitted, he or she must stay in a "clean" area in the hospital and the hospitalization time must be minimized (e.g., ambulatory paracentesis and outpatients albumin infusions can be performed).

Endoscopic exams, due to the extensive production of aerosols involved, are a high risk for the spread of SARSCov2, so they should only be performed in urgent cases. In the context of hepatology, the 2 main indications are upper GI bleeding, with particular attention to any related to portal hypertension or bile duct obstruction. The strategy for eradicating esophageal varices after an episode of rupture is not defined and should be considered on a case basis. Endoscopic examinations for the stratification of portal hypertension should be postponed. It may be decided to start nonselective $\beta$-blockers, according to the Baveno VI criteria, without endoscopy.

Liver biopsy should be deferred when not essential. Follow-up of liver patients will be increased when collaboration with primary care doctors is possible [28, 29].

However, cirrhosis is a disease with inexorable evolution, so the need to focus all efforts on responding to the pandemic has consequences for these patients in the short, medium, and long term.

In the short term, patient follow-up can be modified, i.e., seeing that there are fewer appointments and assessments and, potentially, crucial decisions can be delayed, such as the introduction of prophylactic therapy for the rupture of esophageal varices or the indication of resection or transplantation in cases of malignant liver nodules. A reduction of around $25 \%$ of donors has already been identified in this first quarter of the pandemic, and also a reduction in the number of liver transplants.

In the medium term, when the social-distancing measures are withdrawn or reduced, it is expected that 3 problems will arise. The first is the identification of seriously ill patients and those who may have far exceeded the optimal time point for treatment decisions about a biologically more aggressive disease which evolves without adequate surveillance during the entire phase of mitigation. Second, during the pandemic, there has been an increase in the consumption of alcohol and opioids. More people may develop liver disease and will appear as new patients, 
thus increasing the disease burden $[30,31]$. The third problem relates to institutions and health professionals, who, also exhausted, will have to respond to an excessive number of patients in a short time, and their capacity to respond will likely be exceeded [32].

In the long term, irregular follow-up will be reflected in the health metrics, with an increase in the number of decompensations, HCC, patients on transplantation lists, and mortality [33]. Modulation studies of SARS-Cov2 infection suggest that the pandemic will not end abruptly, but that there will be periods of COVID-19 activity in the coming years [34]. We must prepare ourselves clinically and socially for the outcomes for liver patients as a consequence of changes in their follow-up due to COVID-19.

There is a huge gap in knowledge regarding SARSCov2/COVID-19 and the relationship of both the virus and the disease to liver disease, concerning its different etiological aspects and different clinical forms. It is extremely important to acquire this knowledge in the present moment and for the future. The Associação Portuguesa para o Estudo do Fígado (APEF; Portuguese Association for the Study of the Liver) supports all initiatives that favor the acquisition of this knowledge.

\section{Statement of Ethics}

This work does not require evaluation by an Ethics committee. The authors declare that they have complied with the principles of ethics and the absence of plagiarism.

\section{Disclosure Statement}

The authors declare no conflicts of interest of any kind regarding this manuscript.

Funding Sources

There was no funding.

\section{Author Contributions}

All authors participated in the conception, writing and revision of the manuscript. The final version was approved by the authors.

\section{References}

1 Cheng VC, Lau SK, Woo PC, Yuen KY. Severe acute respiratory syndrome coronavirus as an agent of emerging and reemerging infection. Clin Microbiol Rev. 2007 Oct;20(4): $660-94$.

2 Jin X, Lian J-S, Hu J-H, et al. Epidemiological, clinical and virological characteristics of 74 cases of coronavirus-infected disease 2019 (COVID-19) with gastrointestinal symptoms. Gut. 2020;0:1-8.

3 Zhan M, Qin Y, Xue X, Zhu S. Death from Covid-19 of 23 Health Care Workers in China. N Engl J Med. 2020 Apr. DOI: doi: 10.1056/NEJMc2005696.

$4 \mathrm{Wu}$ Z, McGoogan JM. Characteristics of and Important Lessons from the Coronavirus Disease 2019 (COVID-19) Outbreak in China: Summary of a Report of 72314 Cases from the Chinese Center for Disease Control and Prevention. JAMA. 2020 Feb;323(13):1239.

5 Morty RE, Ziebuhr J. Call for Papers: the pathophysiology of COVID-19 and SARSCoV-2 infection. Am J Physiol Lung Cell Mol Physiol. 2020 Apr. DOI: 10.1152/ajplung.00136.2020.

6 Abassi Z, Assady S, Khoury EE, Heyman SN Letter to the Editor: Angiotensin-converting enzyme 2: an ally or a Trojan horse? Implications to SARS-CoV-2-related cardiovascular complications. Am J Physiol Heart Circ Physiol. 2020 May;318(5):H1080-3.
7 Ng SC, Tilg H. COVID-19 and the gastrointestinal tract: more than meets the eye. Gut. 2020 Apr. DOI: 10.1136/gutjnl-2020-321195.

8 Sutton D, Fuchs K, D'Alton M, Goffman D. Universal Screening for SARS-CoV-2 in Women Admitted for Delivery. N Engl J Med. 2020 Apr. DOI: 10.1056/ NEJMc2009316.

9 https://covid19.min-saude.pt/wp-content/uploads/2020/04/45_DGS_boletim_20200416FINAL.pdf

10 Jin YH, Cai L, Cheng ZS, Cheng H, Deng T, Fan YP, et al.; for the Zhongnan Hospital of Wuhan University Novel Coronavirus Management and Research Team, Evidence-Based Medicine Chapter of China International Exchange and Promotive Association for Medi$\mathrm{cal}$ and Health Care (CPAM). A rapid advice guideline for the diagnosis and treatment of 2019 novel coronavirus (2019-nCoV) infected pneumonia (standard version). Mil Med Res. 2020 Feb;7(1):4.

11 Jin X, Lian JS, Hu JH, Gao J, Zheng L, Zhang YM, et al. Epidemiological, clinical and virological characteristics of 74 cases of coronavirus-infected disease 2019 (COVID-19) with gastrointestinal symptoms. Gut. 2020 Mar;0: $1-8$.

12 Cai Q, Huang D, Yu H, et al. Characteristics of Liver Tests in COVID-19 Patients. J Hepatol. 2020 Apr 13. pii: S0168-8278(20)30218-X.
13 Chen N, Zhou M, Dong X, Qu J, Gong F, Han Y, et al. Epidemiological and clinical characteristics of 99 cases of 2019 novel coronavirus pneumonia in Wuhan, China: a descriptive study. Lancet. 2020 Feb; 395(10223):507-13.

14 Zhang C, Shi L, Wang FS. Liver injury in COVID-19: management and challenges. Lancet Gastroenterol Hepatol. 2020 May;5(5):42830.

15 Guan WJ, Ni ZY, Hu Y, Liang WH, Ou CQ, He JX, et al.; China Medical Treatment Expert Group for Covid-19. Clinical characteristics of coronavirus disease 2019 in China. N Engl J Med. 2020 Feb. DOI: 10.1056/NEJMoa2002032.

16 Sun J, Aghemo A, Forner A, Valenti L. COVID-19 and liver disease. Liver Int. 2020 Apr. DOI: $10.1111 /$ liv.14470.

17 Asrani SK, Devarbhavi H, Eaton J, Kamath PS. Burden of liver diseases in the world. J Hepatol. 2019 Jan;70(1):151-71.

18 Ji D, Qin E, Xu J et al. Implication of non-alcoholic fatty liver diseases (NAFLD) in patients with COVID-19: a preliminary analysis. J Hepatol. 2020 Apr. DOI: 10.1016/j. jhep.2020.03.044.

19 D’Antiga L. Coronaviruses and immunosuppressed patients. The facts during the third epidemic. Liver Transpl. 2020 Mar. DOI: 10.1002/lt.25756 
20 Yang Z, Liu J, Zhou Y et al. The effect of corticosteroid treatment on patients with coronavirus infection: a systematic review and meta-analysis. J Infect. 2020 Apr. DOI: 10.1016/j.jinf.2020.03.062

21 Li X1, Xu S1, Yu M1, et al.Risk factors for severity and mortality in adult COVID-19 inpatients in Wuhan. J Allergy Clin Immunol. 2020 Apr. DOI: 10.1016/j.jaci.2020.04.006.

22 Lleo A, Invernizzi P, Lohse AW et al. Highlights for management of patients with Autoimmune Liver Disease during COVID-19 pandemia. J Hepatol. 2020 Apr. DOI: 10.1016/j.jhep.2020.04.002.

23 Xu L, Liu J, Lu M, Yang D, Zheng X. Liver injury during highly pathogenic human coronavirus infections. Liver Int. 2020 Mar. DOI: 10.1111/liv.14435.

24 Li J, Fan JG. Characteristics and Mechanism of Liver Injury in 2019 Coronavirus Disease. J Clin Transl Hepatol. 2020 Mar;8(1):13-7.
25 Bangash MN, Patel J, Parekh DCOVID-19 and the liver: little cause for concern. Lancet Gastroenterol Hepatol. 2020 Mar. DOI: 10.1016/S2468-1253(20)30084-4.

$26 \mathrm{Li} \mathrm{X}, \mathrm{Xu} \mathrm{S}, \mathrm{Yu} \mathrm{M}$, et al. Risk factors for severity and mortality in adult COVID-19 inpatients in Wuhan. J Allergy Clin Immunol. 2020 Apr. DOI: 10.1016/j.jaci.2020.04.006

27 Fernández-Ruiz M, Andrés A, Loinaz C, Delgado JF, López-Medrano F, San Juan R, et al. COVID-19 in solid organ transplant recipients: a single-center case series from Spain. Am J Transplant. 2020 Apr. DOI: 10.1111/ ajt.15929.

28 Fix OK, Hameed B, Fontana RJ, Kwok RM, McGuire BM, Mulligan DC, et al. Clinical Best Practice Advice for Hepatology and Liver Transplant Providers During the COVID-19 Pandemic: AASLD Expert Panel Consensus Statement. Hepatology. 2020 Apr. DOI: 10.1002/hep. 31281

29 Boettler T, Newsome PN, Mondelli MU, Maticic M, Cordero E, Cornberg M, et al. Care of patients with liver disease during the COVID-19 pandemic: EASL-ESCMID position paper. JHEP Rep. 2020 Jun;2(3):100113.
30 Clay JM, Parker MO. Alcohol use and misuse during the COVID-19 pandemic: a potential public health crisis? Lancet Public Health. 2020 Apr. DOI: 10.1016/S2468-2667 (20)30088-8.

31 Sun Y, Bao Y, Kosten T, Strang J, Shi J, Lu L. Editorial: Challenges to Opioid Use Disorders during COVID-19. Am J Addict. 2020;29(3): 174-5.

32 Castelletti S. A Shift on the Front Line. N Engl J Med. 2020 Apr. DOI: 10.1056/ NEJMp2007028.

33 Tapper EB, Asrani SK. COVID-19 pandemic will have a long-lasting impact on the quality of cirrhosis care. J Hepatol. 2020 Apr. DOI: 10.1016/j.jhep.2020.04.005.

34 Kissler SM, Tedijanto C, Goldstein E, Grad YH, Lipsitch M. Projecting the transmission dynamics of SARS-CoV-2 through the postpandemic period. Science. 2020 Apr. 10.1126/ science.abb5793. 